\title{
Das Sterben erinnern Erzählungen aus Angehörigenperspektive
}

Michaela Thönnes

"When disruption is perceived it must be explained, and narratives provide a framework." Peter L. Callero, The Sociology of Self ${ }^{1}$

\section{Einleitung}

Die Besonderheiten des Erzählens vom Sterben entstehen aufgrund der Intimität des Erinnerns eines signifikanten Verlusts und der Grenzerfahrung, die das Sterben einer nahestehenden Person für Hinterbliebene bedeutet. Angehörige zu diesem sensiblen Erfahrungsbereich zu befragen, bedeutet, die Verantwortung für die Konsequenzen dieses intimen Forschungssettings übernehmen $\mathrm{zu}$ wollen. Forschende gestalten eine Interviewsituation einerseits durch eine dem persönlichen Dialog verwandte Atmosphäre. Andererseits ist jedoch eine dem persönlichem Dialog angemessene tatsächliche Gleichberechtigung ${ }^{2}$ zwischen den Interviewpartnern ausgeschlossen durch das dem Interview eigene themenspezifische Frage-Antwort-Setting mit der daran anschliessenden Veröffentlichung von Interviewinhalten.

Die im Rahmen des vorliegenden Beitrags analysierten Interviews, die Erfahrungen hinsichtlich der Rekrutierung von Teilnehmerinnen und Teilnehmern sowie die konkreten Erlebnisse emotionaler Ausnahmesituationen auf Seiten der Befragten bestätigen den methodisch und ethisch besonders anspruchsvollen Aspekt der Forschungskonzeption. Die geringe Anzahl von Studien über den als sozial definierten Sterbeprozess und die bisher offen gebliebenen Antworten auf Fragen, wie wir als Gesellschaft und Individuen Sterbende in Zukunft begleiten können, erhöht die Intimität des Sterbens und weckt zugleich das (soziologische) Forschungsinteresse. Sterben ist kein rein organischer oder psychischer Prozess. Der

1 Peter L. Callero, The Sociology of Self, in: Annual Review of Sociology 29 (2003), 115-133, 124.

2 Steinar Kvale, Dominance Through Interviews and Dialogues, in: Qualitative Inquiry 12 (2006), 480-500. 
gesellschaftliche Diskurs zur Sterbewirklichkeit befasst sich aber in erster Linie mit Fragen, wie mit der Bevölkerungsentwicklung umzugehen sei. Es werden Zukunftsszenarien entwickelt, in denen überwiegend alte, demente und körperlich schwache Menschen das Gesundheitssystem und die Gesellschaft vor ökonomische Probleme stellen. Die aktuelle Sterbewirklichkeit und die darin notwendige Rolle der Gesellschaft beleuchtet dieser Diskurs dagegen nur schwach.

Ja, Sterbewirklichkeit ist hauptsächlich geprägt von der Hilfsbedürftigkeit und Selbstbestimmung, die Sterbende und die für sie verantwortlichen Mitmenschen erfahren oder erwirken können. Doch mit der Reflexion möglicher Handlungsfelder im Sterbeprozess wirken soziale Bedingungen einer gesellschaftlich legitimierenden Sterbekultur auf das Sterben Einzelner. Es macht für Sterbende und ihre Sterbebegleitung einen Unterschied, ob sie auf sozial geschaffene Strukturen eines Hospiz- oder Palliativnetzwerks und deren Informations- und professionelle Betreuungsangebote zurückgreifen können oder nicht. Zu bedenken ist, dass die Besonderheit des Sterbens begründet liegt in der mit der physiologischpsychologischen Defizitentwicklung einhergehenden Veränderung der sozialen Rolle und des sozialen Status Sterbender selbst und derer, die Sterbende begleiten. ${ }^{3}$ Im Folgenden werden soziologisch relevante Facetten des Sterbens auf der Grundlage von Erzählungen Angehöriger beleuchtet, die sich an die Sterbebegleitung einer ihnen nahestehenden Person erinnern.

\section{Methode}

Die Studie wurde als qualitative Studie angelegt, um Datenmaterial zu generieren, das Aussagen über erlebte Sterbeprozesse zulässt. Qualitative Sozialforschung folgt dem Postulat, sozialwissenschaftliche Forschung solle ihren Ausgang in der Wirklichkeitskonzeption der Handelnden nehmen. Im Vordergrund steht das Nachvollziehen des Interpretationsprozesses von Befragten, in dessen Verlauf sie ihren Wahrnehmungen in Bezug auf die befragte Handlungssituation sowie sich selbst als Handelnden Bedeutungen zuschreiben. Für den vorliegenden Artikel wurden Interviews mit Angehörigen analysiert, die selbst sterbende Angehörige begleitet hatten. Diese waren als

3 Allan Kellehear, A Social History of Dying, Cambridge 2007. 
leitfadengestützte, problemzentrierte Interviews ${ }^{4}$ konzipiert und wurden aufgrund der zu erwartenden Emotionalität ${ }^{5}$ des Befragungsszenarios für die Methode des narrativen Interviews ${ }^{6}$ geöffnet.

Die Befragten ${ }^{7}$ wurden mittels eines Schneeballverfahrens und anhand eines Telefonanrufs sowie der Zusendung eines Fragebogens vor dem Interview kontaktiert, um sie über den Zweck des Interviews als Teil einer soziologischen Studie zum Sterbeort ${ }^{8}$ zu informieren. Die gewählte Methode exploriert die in einer Gesellschaft plural vorliegenden Individualerfahrungen. Diese für die Erhebungsmethode des problemzentrierten und narrativen Interviews typische Gegenstandsangemessenheit ermöglicht, die Interaktions-, Sinn- und Beziehungsstrukturen der Befragten auf verschiedenen Analyseebenen im Erzählen über das Sterben zu interpretieren. Das problemzentrierte oder narrative Interview ist gegenüber anderen Befragungsmethoden im Vorteil, wenn in schwach erforschten Themenbereichen sozialer Lebenswelten die jeweiligen Formen der Sinnkonstruktionen der Befragten wahrzunehmen sind. Die größtmögliche Offenheit in der Interviewführung bietet dafür eine ausgezeichnete Voraussetzung. Das narrative Interview gibt den Befragten die Möglichkeit zur Selbst- und Verhältnisreflexion, und die Interviewer können schrittweise ein adäquates Verständnis der Problemsicht der Befragten entwickeln.

Das im Folgenden ausgewählte Interviewmaterial wurde ursprünglich im Rahmen einer Arbeit gewonnen, das der Analyse allgemeiner Einstellungen gegenüber dem Sterben und den Sterbeorten sowie den subjektiven Dispositionen wie zum Beispiel Wün-

\footnotetext{
4 Andreas Witzel, Verfahren der qualitativen Sozialforschung. Überblick und Alternativen, Frankfurt a.M. 1982.

5 Gabriele Rosenthal, Biographisch-narrative Gesprächsführung: Zu den Bedingungen heilsamen Erzählens im Forschungs- und Beratungskontext, Psychotherapie und Sozialwissenschaften, in: Zeitschrift für qualitative Forschung 4 (2002), 204-227.

6 Fritz Schütze, Biographieforschung und Narratives Interview, in: Neue Praxis 13/3 (1983), 283-293.

7 Die Befragten waren vier Männer und vier Frauen im Alter von 24 bis 70 Jahren. Sie lebten in Nordrhein-Westfalen (D), Rheinland-Pfalz (D) und der Schweiz. Die Verstorbenen (zwei Männer im Alter von 33 und 45 Jahren, sechs Frauen im Alter von 69 bis 96 Jahren) verstarben in Krankenhäusern, Altenheimen oder zuhause in mittelgroßen Städten Nordrhein-Westfalens oder in kleinen Gemeinden in Rheinland-Pfalz. Sie wurden von den Verwandten, von Pflegepersonal wie auch in Mischformen begleitet und versorgt.

8 Michaela Thönnes, Sterbeorte in Deutschland. Eine soziologische Studie, Frankfurt a.M. 2013. Michaela Thönnes/Nina Jakoby, Wo sterben Menschen? Zur Frage des Sterbens in Institutionen, in: Zeitschrift für Gerontologie und Geriatrie 44/5 (2011), 336-339.
} 
schen, Vorlieben, Hoffnungen, Ängsten, Abneigungen, Intentionen und Plänen der Befragten diente. Die für den vorliegenden Artikel gewählte Analyse befasst sich hingegen mit der Fragestellung, wie Angehörige von ihren Erlebnissen mit Sterbenden und dem Sterbeprozess erzählen. Die Analyse der Interviews bezieht sich auf die unterschiedlichen Sprech- und Gesprächsstile der Befragten wie z.B. deren Reaktion auf die Eingangsfrage. Auch wirkt die in der Sterbesituation seitens der Angehörigen unterschiedlich erlebte Kommunikation mit medizinischem Personal in der Interviewsituation durch unterschiedliche Themenschwerpunktsetzung im Erzählfluss noch immer kontrastierend nach. Gleichzeitig finden sich mit der Tendenz zur Entkörperlichung sterberelevanter Beschreibungen oder der besonderen Hervorhebung der Persönlichkeitsmerkmale der Verstorbenen in den Interviews Gemeinsamkeiten der Sterbeerzählstile.

Eine Besonderheit von Interviews über das Sterben und des damit verbundenen Feldzugangs zeigt sich gleich zu Beginn der Studie, welche die beschriebene Intimität von Sterbeerlebnissen bestätigt. Das gewählte Schneeballverfahren erweist sich als geeignete Methode, da der Zugang zu den Befragungspersonen ein spezifisches Merkmal aufweist: Die Befragten sind nur bereit, ein Gespräch über ihre Sterbeerfahrung zu führen, weil das Interview durch Angehörige, Freundinnen und Freunde und Bekannte - also der Interviewerin wie den Interviewten vertraute Personen - vermittelt und damit der Wunsch nach Rücksichtnahme auf die besondere Intimität dieses Themenfelds gewährleistet wurde. Exemplarisch kann die Sensibilität im Forschungsumfeld Sterben anhand des folgenden Zitats verdeutlicht werden: »Ich sag Ihnen mal so. Im Grunde, wenn Sie jetzt nicht den Kontakt über (Namen) hergestellt hätten, dann hätten Sie bei mir keinen Fuß zwischen die Tür gekriegt." (Interview 3, S. 5/25)

Die Bereitschaft der Befragten, den Anspruch auf Intimität zugunsten einer Teilnahme an den Interviews ausnahmsweise zurückzustellen, wurde in den Vorgesprächen und beim telefonischen Erstkontakt begründet. Die Befragten wollten die Gelegenheit nutzen, ihren Beitrag zu einem gesellschaftlich relevanten Thema zu leisten. Sie erachteten es als notwendig, dass wissenschaftliche Forschung die Kommunikation über das schwierige Themenfeld des Sterbens voranbringt. 


\section{Sterbeerzählungen von Angehörigen}

Im folgenden Abschnitt werden Beobachtungen zu Sprech- und Gesprächsstilen wiedergegeben, die im Verlauf der jeweiligen Interviews auffielen. Die erste Reaktion der Befragten auf die Eröffnungsfrage der Interviewerin gibt Hinweise auf die kommunikative Zugänglichkeit des Themenbereichs Sterben für die Befragten und auf die Ordnungsprinzipien ihrer Erinnerungen. Die erste Analyseebene legt dar, wie die Befragten explizit auf den Sterbeprozess zu sprechen kommen und ob oder wie sie diesen im Verlauf des Interviews beschreiben (3.1). Die zweite und die dritte Analyseebene befassen sich mit den beobachteten Unterschieden (3.2) und Gemeinsamkeiten der Sterbeerzählungen (3.3).

\subsection{Sprech- und Gesprächsstile in der Reaktion auf die Eröffnungsfrage}

Die Analyse der Interviews beginnt mit der Frage, wie sich das Antwortverhalten anhand eines offen formulierten Erzählstimulus zur Sterbeerinnerung entwickelt. Die nachfolgenden Interviewausschnitte führen die unterschiedlichen Antworten der Befragten auf, die diese nach einer kurzen Begrüßung und Eröffnungsphase zur ersten Frage nach dem Sterbeerlebnis äußerten. Die Eröffnungsfrage lautete:

"Ich möchte mit Ihnen heute über das Thema Tod und Sterben sprechen und Sie zu Anfang unseres Gesprächs einfach bitten, mir zu erzählen, wie Sie das Sterben und den Tod der Verwandten oder Nahestehenden erlebt haben, also um wen es ging, was passiert ist."

In dieser Eröffnungsphase offenbaren sich drei Antwortstile: (1.) die Umorientierung bzw. Ablenkung, (2.) der direkte Einstieg ohne Eröffnungsfrage und (3.) die Antwort auf die Eröffnungsfrage.

\subsubsection{Umorientierung und Ablenkung nach der Eröffnungsfrage}

Dieser Antwortstil zeichnet sich durch Rückfragen der Befragten zu Themen aus, die außerhalb der Inhaltsebene oder der Frage selbst stehen. Dabei lassen sich zwei Foki unterscheiden. Der erste Fokus der Befragten richtet sich auf technische Aspekte der Interviewsituation. Eine Ablenkung von der Eröffnungsfrage entsteht durch die Aufmerksamkeitsverlagerung auf das Aufnahmegerät und dessen Funktionsfähigkeit. "Noch eine Frage zur Technik, sind Sie 
sicher, dass das gut aufnimmt? [...]: Dann brauchen Sie also keine Probeaufnahme? (Interview 2, S. 2/33). Ein zweiter Fokus kann als Frage- und Intervieworientierung interpretiert werden. Vor einer genaueren und längeren Antwortphase kommt es seitens der Befragten zu den folgenden thematischen Rückversicherungen: "Es geht um jemanden, der mir nahesteht, bei dem ich selbst beim Sterben dabei war? Verstehe ich das richtig?" (Interview 4, S. 2/42); "Was ich zu erzählen habe?» (Interview 6, S. 1/29); »Was mir wichtig war?» (Interview 7, S. 1/30).

Diese Rückfragen sind als rhetorisch zu verstehen und lassen keine direkten Rückschlüsse auf eine themenspezifische Reaktion zu. Doch dienen solche Fragen in der Regel der gegenseitigen Vergewisserung, dass das nun Gesagte von besonderer Bedeutung sein wird. Die kurze durch die Rückfrage geschaffene Bedenkzeit kennzeichnet die Beantwortung als wichtig oder besonders bewusst.

\subsubsection{Direkter Einstieg ohne Eröffnungsfrage}

Im Prozess der gegenseitigen Begrüßung wird, ohne einleitend dazu aufgefordert zu werden, direkt vom Sterben erzählt, bevor die Interviewerin die Gelegenheit hatte, über Aufnahmetechnik und Anonymisierung des Interviews zu informieren sowie die eröffnende Frage zu stellen. Über das soziale Umfeld, den Sterbeort und die Sterbesituation wird ohne Umschweife erzählt, wie die folgenden Interviewausschnitte verdeutlichen.

"Da waren wir gewesen, da war meine Schwester dabei, die Nichte also die Tochter von meiner Schwester, wir hatten mal zu dritt ein gutes Gespräch gehabt, die Mutter hat nicht mehr so viel mitgekriegt. Sie hat also in derVergangenheit so ein bisschen gelebt, aber hat ... was da ist, immer noch wahrgenommen, die Stimmen direkt eingeordnet: `Bist du es? A Auch wenn, ..., das war also bei ihr vorhanden alles. Wir hatten noch fast zwei Stunden im Altenheim, sie war fünf Jahre im Altenheim, da waren wir fast zwei Stunden bei ihr, nachher, gewesen. Ja. War das auch das letzte Mal. Dann bei ihr verabschiedet, ja und dann ist sie am nächsten Tag, also abends spät, nachts, ist sie dann gestorben.« (Interview 3, S. 1/25)

Für den Befragten dieses Interviews ist es wichtig, gleich zu Beginn der Begegnung mit der Interviewerin die soziale und individuelle Situation der Verstorbenen am Lebensende zu klären. Indem er in dieser ersten Phase wie auch später wiederholend die mit der Sterbenden geführten Gespräche beschreibt, hebt er diese für ihn 
wichtige Facette des Sterbeprozesses besonders hervor. Die Sterbende wurde vom kleinen Personenkreis der Unterstützer trotz ihrer altersbedingten kognitiven Einschränkung sowie langwieriger physiologischer und psychologischer Zerfallsprozesse nicht von den für sie relevanten Entscheidungsfindungsprozessen ausgeschlossen. Der Befragte dieses Interviews hatte seine Mutter im Altenheim begleitet. Die Befragte des folgenden Interviewausschnitts hatte ihren Sohn ohne ambulante Palliativversorgung zuhause gepflegt.

"Ja, ein interessantes Thema: `Das betrifft ja mich nicht $\iota$, ne, >das betrifft ja die anderen. [...] Aber wir beide, mein Mann und ich, haben uns ganz genau überlegt, ob der eine sich dem anderen zumuten kann, wenn dann einer in eine ganz schwierige Situation kommt. Und die wird kommen, das ist ja so sicher wie das Amen in der Kirche. Dann weiß ich ganz genau, dass er es nie leisten könnte, mich zu versorgen, und dann habe ich gesagt: >(Name), und dann gehe ich ins Hospiz und dann kannst $\mathrm{Du}$ jeden Abend nachhause gehen, in deinem Bett schlafen, wenn du willst und umgekehrt genauso. [...] Und dann kann das Sterben auch im Hospiz stattfinden. Also mit unserem Sohn hat alles zuhause stattgefunden. Aber das war ein, ein, ein ganz besonderer Verlauf der Erkrankung, das war ein (Erkrankung), das war gnadenlos, der Verlauf. (Kleine Pause) Der war schon sinnlos, von Anfang an. Aber wir haben natürlich alle geglaubt, er schafft es. [...] Und ich dachte: ^Aber mit 10\%, da wird doch der (Namen) mit fertig. Das ist doch ein Klacks`, aber das war sein Todesurteil. Der Tumor hat sich gar nicht beeindrucken lassen durch, weder durch Bestrahlung noch durch Chemo. Das ist im (Körperteil) hochgewachsen, und er war zum Schluss (Körperregion) gelähmt.Aber wir haben das zuhause geschafft. [...] Und das war sein Wunsch: `Eltern, ich möchte zuhause sterben', und wir haben ihm das ermöglicht. Aber ich weiß, was das für eine schwere Aufgabe war.« (Interview 5, S. 1-2/28)

In diesem Interview wird zuerst die verdrängende und doch nachvollziehbare Sichtweise Anderer angesprochen. Dies impliziert das Problem mangelnder Kommunikationsmöglichkeiten von Sterbebegleitern, die erleben, dass die Erfahrungen, von denen Gesprächspartner im Allgemeinen den Eindruck haben, sie beträfen sie nicht, auch schwer zu kommunizieren sind. Dass die Befragte dies direkt zu Beginn des Interviews in Verbindung mit den Schilderungen ihrer eigenen Sterbekommunikation und ihres Sterbeerlebnisses anspricht, dient derVeranschaulichung, für wie alltagsrelevant sie die 
Kommunikation über das Sterben hält. Sie spricht über die Schwierigkeiten, die durch mangelnde Kommunikation und fehlendes Wissen potenziert würden.

\subsubsection{Antwort auf die Eröffnungsfrage}

Ein dritter Antwortstil entspricht dem geplanten Ablauf der Interviewführung. Die Befragten warten nach einer kurzen Begrüßungsphase die Intervieweröffnung und erste Frage $a b$, um auf diese adäquat antworten zu können.

Frage: "Und jetzt wollte ich nur fragen, wer gestorben ist, wer das war, wo das war und was Dir dazu einfällt. Dass Du einfach einmal davon anfängst zu erzählen.»

Antwort: "Ja, also es ist, glaube ich, ziemlich genau fünf Jahre her, dass meine Mutter gestorben ist. Sie war 80 Jahre alt. Und hatte (überlegt kurz), sie hatte sich auf eigenen Wunsch einer Operation unterzogen, mit der sie offenbar ihre Leistungsfähigkeit überschritten hat. Jedenfalls ist sie nach dieser Operation nicht mehr wirklich ins Leben zurückgekehrt. Es war die Sache, ..., sie hat Tage dazwischen gehabt, in der sie vermutlich, ..., sie hat einen Schlaganfall erlitten und hatte dann etwa noch drei Monate gelebt, bis sie gestorben ist. Das war in (Ort), etwas genauer in (Ort) im Krankenhaus, in einem kirchlich geführten Krankenhaus, was für mich erstaunlicherweise, also mich erstaunte es, was das für eine wichtige Dimension ist. Ich habe mit, ja, vor 10 Jahren habe ich mich von der Kirche abgewandt, und von Religion, ohne, ohne aus dem Blick zu lassen, dass die Kirche ein wichtiger Arbeitgeber ist, dass Religion eine wichtige Instanz ist für, für viele Menschen ist, dass kirchliche Instanz eine Hilfeinstanz ist. Nur für mich persönlich gibt es da keineVerbindung. Ich glaube, für meine Mutter war das genau die richtige Instanz, sie war religiös gebunden gewesen. Sie war auch, nicht übermäßig, aber so mit einem Grundverständnis, dass die Kirche ihr weiterhilft." (Interview 8, S. 1-2/25)

Wie in den Antwortpassagen der vorherigen Beispiele zeigt sich direkt zu Beginn des Interviews mit der Vielfältigkeit aufgeführter Themen und einem stockenden Redefluss die Komplexität des Erzählens vom Sterben. Die Befragten sprechen das soziale Umfeld der Sterbenden, Zeit, Bewusstsein, Kommunikation, physiologische Voraussetzungen, Sterbebeginn, Sterbeverlauf, medizinischen Kontext, Religiosität, Sterbeort etc. sofort in ihrer ersten Antwortpassage an. 
Auffällig ist, dass Erzählungen zum eigentlichen Sterbeprozess nur auf wiederholte Nachfrage im weiteren Gesprächsverlauf entstehen konnten; physiologische und psychologische Defizitentwicklungen der Sterbenden und deren Wirkung auf die Sterbebegleitung bleiben in den Beschreibungen dagegen aus.

Antwort: "Jetzt weiß ich nicht mehr, was Sie gefragt hatten.»

Frage: "Wie Sie den Sterbeprozess erlebt haben? Erzählen Sie einfach, was Ihnen einfallt dazu.»

Antwort: "aja. Dann, ehm, ehm, Anfang (Zeit) konnte sie nicht mehr zuhause leben. Und da haben wir überlegt, wo ist es für sie am besten wäre, in (Ort), das war uns wichtig, zu leben. Sie konnte da noch einigermaßen sich bewegen, ohne Rollstuhl und ohne Rollator. Und da war ein Haus in (Ort), den Namen den nenn ich jetzt: (Namen) Seniorenzentrum. [...] Das wurde umgebaut, (Träger der Pflegeeinrichtung) hat's umgebaut. Es war sehr schön, alles neu und ziemlich nobel und wir haben einen Platz gefunden für sie. [...] In dem, vom (Zeit) bis zum (Zeit), da starb sie. Sie fühlte sich da wohl, sie wurde beachtet, immer super gekleidet, da legte sie Wert und wir legten Wert drauf. Das Zimmer war immer mit Blumen versehen. Und ehm, sie war bei den Mitbewohnern beliebt, auch bei den Männern. Es entwickelten sich kleine Freundschaften, in Anführungszeichen. Ganz reizend in dem Alter. Ja und dann der engere Prozess des Sterbens, der pfff, das war, ein halbes Jahr vor dem Tod fing das eigentlich an. Die Demenz nahm zu, von (Zeit) an, so langsam aber sicher (S. 3/33). [...] Sie ist eigentlich so langsam, das hab' ich auch so geschrieben, in dem ersten Fragebogen, aus Altersschwäche, so peu à peu gestorben. Wunderbar, wunderschön eigentlich, bis zum Schluss." (Interview 2, S. 7/33)

Die Befragten erzählen retroperspektiv von dem für sie subjektiv Besonderen des Sterbeprozesses. Die wiederkehrende Ablenkung von der Frage nach dem Sterben hin zu einer Persönlichkeits- und allgemeinen Lebensweltbeschreibung der sozialen Sterbebedingungen ist ein typisches Muster des beobachteten Erzählablaufs im Interview. 


\subsection{Kontrastierende Sterbeerzählungen}

Die jeweils eröffnenden Erzählungen der Interviewten in den Interviews 6, 7, 8 und 9 weisen unterschiedliche Schwerpunkte in Bezug auf die zu Beginn als besonders wichtig eingeschätzten Inhalte auf. Die Kommunikationserfahrungen im Sterbeprozess nehmen dabei einen besonderen Stellenwert ein.

Die Befragte des Interviews 6 stellt die Tiefe des Schocks, der bei ihr und der Mutter durch den Zusammenbruch und das anschließende Koma des Verstorbenen ausgelöst wird, sowie die fehlende Kommunikation zwischen dem Ärztepersonal und ihnen als Angehörigen ins Zentrum der Erzählung. ")a, und dann haben die Ärzte versucht, uns davon zu überzeugen, dass wir meinenVater doch ins Pflegeheim geben sollten. Und der Oberarzt meinte dann: »Der bekommt doch sowieso nichts mehr mit." (Interview 6, S. 7/29)

Im Interview 7 steht die von Vater, Bruder und der Verstorbenen selbst verschwiegene Information über die Schwere der Krankheit im Vordergrund - und der daraus für den Befragten resultierende Verlust der Einflussnahme auf eine adäquate Versorgung der Verstorbenen:

" $[\mathrm{U}]$ nd es ist so gewesen, dass sie lange vorher Unterleibskrebs hatte, das ist in der Familie nicht erzählt worden, [...] und dann gab's eine Chemo, das ist das erste Mal, $[\ldots]$ und dann habe ich überhaupt zum ersten Mal erfahren, dass meine Mutter krank ist. Das ist nie erzählt worden. [...] Das ist von ihr und meinem Vater, wurde mir das nicht erzählt.« (Interview 7, S.1-2/30)

Die Befragten der Interviews 8 und 9 wählen einen gegenüber den Interviews 6 und 7 verschiedenen Ausgangspunkt ihrer Erzählung. Sie betrachten die Situation des Sterbeprozesses in Hinsicht auf das, was dem Verstorbenen besonders in der Sterbesituation von Bedeutung gewesen sein könnte. Beide Verstorbenen (Interview 8 und 9) werden zu Beginn als Persönlichkeiten mit den für sie wichtigen Werten wie Religiosität und Arbeitshaltung vorgestellt (vgl. Interviewausschnitte S. 7, 11 und 12). Die Sterbeerfahrungen der Befragten der Interviews 6 und 7 sind hingegen sowohl durch die ausgeprägte Veränderung der Rolle der Eltern und deren dramatischen Sterbeverlauf sowie durch insgesamt schwierige und konfliktreiche Kommunikationserlebnisse im Umfeld der Sterbenden geprägt.Vor diesem Hintergrund ist nachvollziehbar, dass sich eine Eröffnung der Gespräche hierüber bei den Befragten der Interviews 6 und 7 gegenüber den Interviews 8 und 9 unterscheidet: Zwar wurden alle Befragten in ihrem Alltag plötzlich durch eine Diagnose mit Sterbe- 
verläufen konfrontiert. Doch schildern die Befragten des Interviews 6 und 7 die Situation der professionellen Unterstützung sowie Kommunikationserlebnisse im Zusammenhang mit der Sterbebegleitung vollkommen different von denen der Interviews 8 und 9. In den Interviews 6 und 7 dominieren zu Beginn die Beschreibungen des Schocks aufgrund der Diagnose und der durch Unterlassung und Manipulation geprägten Kommunikation unter den Angehörigen oder zwischen Angehörigen und Fachkräften. Die Verstorbenen der Interviews 8 und 9 wurden eng und kommunikativ stark von sterbeerfahrenen medizinisch-pflegerischen Fachkräften begleitet. Beide Befragten erwähnen den Schock einer plötzlichen und todbringenden Diagnose eher in einem Nebenstrang der Erzählung.

Die Angehörigen kommen trotz unterschiedlicher Abfolge und verschiedener Schwerpunkte der Themen im Verlauf der Interviews auf die gleichen Inhalte zu sprechen und gehen dabei stark auf die Beschreibung der Verstorbenen ein. Die Wahl der Inhalte zur Eröffnung spiegelt die Intensität wider, in dem sich die Befragten an die verstorbene Person erinnern; sie hat Einfluss darauf, inwieweit deren emotionale Beziehung "wiederbelebt" wird und auch die erfahrenen Gefühle von Unterstützung oder Ausschluss während der Sterbesituation im Interview aktualisiert werden.

\subsection{Gemeinsamkeiten der Sterbeerzählungen}

Die Auswertungen ergeben weiter, dass die Sterbeerzählungen ungeachtet der individuellen Lebensläufe, Sterbeerfahrungen und Kommunikationsstile der Interviewten in Aufbau und Inhalt Ähnlichkeiten aufweisen. Es lassen sich drei im Folgenden dargestellte Themen identifizieren: Entkörperlichung, Beschreibung der Persönlichkeit der Sterbenden und Umfang und Art erlebter sozialer Unterstützung.

\subsubsection{Entkörperlichung}

Im Rahmen der Befragung von Angehörigen Verstorbener fiel ein durchgängig wiederkehrendes Antwortverhalten auf. Die aus forschungsmethodischen Gründen offen gehaltene Frage zu Sterben und Tod veranlasste Antwortstränge, die sich zuerst über ein mehrseitiges Transkript mit Facetten der Persönlichkeit der Verstorbenen während des Sterbeprozesses sowie Erfahrungen mit dem sozialen Umfeld befassten. Trotz eines informativen Vorgesprächs zum Stellenwert der Interviews im Rahmen einer soziologischen Forschungsarbeit über Sterbeort und Sterbeprozess beschrieben die 
Befragten in den Anfangsphasen der Interviews nicht die physischen Verfallsprozesse. Dies kann als "Entkörperlichung « der Sterbeerzählungen interpretiert werden. Dieses Ergebnis - auch nach wiederholtem Nachfragen zum Sterbeprozess - entspricht nicht einer medizinischen und pflegerischen Beschreibung des Sterbens, die die sterbebedingten körperlichen, psychischen und sozialen Verfallprozesse mit der darin begründeten sozialen Abhängigkeit in den Mittelpunkt stellt.

Eine Ausnahme stellt der folgende Interviewausschnitt dar, in dem die Tochter insbesondere den konkreten körperlichen Verfall und den physiologischen Sterbeprozess des Vaters beschreibt:

"Es war ein schrecklicher Anblick, weil mein Vater ein großer kräftiger Mann war, der immer hart körperlich gearbeitet hat, er wurde halt immer dünner, ausgezehrter, und ihn da so liegen zu sehen, mit Urinbeutel und dem Luftröhrenschnitt und dem Schlauch durch die Nase, das war, war schon komisch irgendwie. So unrealistisch kommt einem das vor. [...] Im (Monat $n$ ) ist er, ist er ins Koma gefallen, im (ein Monat später n) haben wir ihn dann mit nachhause genommen, und am (drei Monate später n) ist er dann gestorben. "(Interview 6, S. 8/29)

\subsubsection{Persönlichkeit der Verstorbenen}

Die Schilderungen des Sterbeerlebnisses werden imVerlauf des Interviews von einer Vorstellung der Persönlichkeit der Verstorbenen zu ihren gesunden Lebzeiten eingeleitet. Diese dient als Referenzpunkt für die Erzählungen über das Lebensende. Die Besonderheiten eines Beginns oder Verlaufs des Sterbeprozesses und deren Bedeutung für die Befragten werden zu der Persönlichkeit der Verstorbenen in Bezug gesetzt.

"Also bei ihr war das Sterben die konsequente Weiterführung des Lebens. (Überlegt) So lange, als sie erkannt hat, dass es, sie ihr Leben nicht mehr selbst bestimmen kann, fand sie es nicht mehr, nicht mehr lebenswert. [...] Meine Mutter hat immer ein, immer ein konsequentes Leben, ja, ein katholisch konsequentes Leben hat sie geführt. Sie hatte ganz klare, ganz klare Maßgaben." (Interview 8, S. 12/25)

"Meine Mutter war immer eine sehr fleißige Frau, die dann so für mich körperlich sehr abgebaut hat, und dann ist sie auch gestürzt." (Interview 9, S. 1/12) 


\subsubsection{Soziale Unterstützung durch Kommunikation}

Ein wichtiges Thema in den Erzählungen aller Befragten sind Erlebnisse von gelungener Kommunikation oder von Kommunikationsproblemen mit anderen im sozialen Kontext des Sterbeprozesses. Diesbezüglich werden das engere eigene und das weitere soziale Umfeld in der erinnerten Sterbebegleitung beschrieben und verglichen.

" $[\mathrm{U}]$ nd dann eh, ein zweiter Punkt ist: /Warum soll ich Besuche machen, wenn sie ja nachher ja nicht mehr weiß, wenn ich da war.< Nicht einmal: /Warum soll ich Besuche machen, wenn ich mich nicht mit ihr unterhalten kann.< Nie im Leben konnte man sich nicht mehr mit ihr unterhalten. Sie konnte nicht mehr antworten. Bis auf die Momente, wo man dachte: Sie ist ja völlig klar, sie kann ja sprechen und doch antworten." (Interview 2, S. $5 / 33)$

Der Befragte des Interviews 2 beschreibt hier die angespannte Beziehung mit weiteren Angehörigen der Verstorbenen. Als Hauptmerkmal des Konflikts nennt er die Fehleinschätzung der Kommunikationsfähigkeit der Verstorbenen durch deren Nichte sowie deren fehlende Kommunikationsfähigkeit und -bereitschaft.

Die Art und Weise, wie Kommunikation verläuft, zeigt die Wertschätzung gegenüber Verstorbenen und hat ebenso Auswirkungen auf Beziehungen außerhalb des Sterbeprozesses. Das Interview 8 ist im Gegensatz zu Interview 2 ein Beispiel gelungener Kommunikation.

"Das war der Situation ganz und gar angemessen. Sehr würdevoll. Also er hat auch uns mit einbezogen. Er hat, um uns zu sagen, was es genau bedeutet, und sich vergewissert, dass wir von dieser Patientenverfügung wissen, und also alle, alle formalen Eckpunkte hat er bestritten. Aber ohne dass Du das Gefühl hattest, der geht jetzt seine Checkliste durch, was er sagen muss. Also das fand ich sehr, sehr würdevoll. Auch, auch gegenüber ihr, als sie, wie heißt das, als sie nicht mehr im Vollbesitz ihrer geistigen Kräfte war, diese nicht abzuerkennen. $\mathrm{Zu}$ sagen: 'Sie hat jetzt gesagt, es ist jetzt soweit‘, und das, das hat mich sehr, sehr bewegt." (Interview 8, S. 8/25)

Interviewte bemaßen die Qualität der Rolle Anderer, von Freunden oder Angehörigen, dem medizinischen Personal oder professionellen Sterbebegleitern im Allgemeinen daran, in welcher Weise diese sich als Kommunikationspartner und -partnerinnen eigneten. Der 
Wunsch der befragten Angehörigen nach Kommunikation ging über die Einschätzung des Nutzens eines Gesprächs hinaus und berührte soziale Handlungsfelder, die den Sterbenden und ihrem sozialen Umfeld durch die Kommunikation ermöglicht würden. Dabei zeichneten die Befragten ein facettenreiches Bild der Voraussetzungen und der Merkmale einer gelungenen Kommunikation. Ob und wie die Befragten über ihr Sterbeerlebnis sprechen konnten, war für alle ein Kernelement einer positiv erinnerten Sterbebegleitung.

"Als seine Tochter ihm angeboten hat, , Wir werden jemanden suchen für dich,Vater, der uns entlastet, und dann hat er gesagt, 'Bloß keine Betschwester! Und dann kam ich da rein und als ich nach dem ersten Gespräch ging, da hat er meine Hände umklammert, und es liefen ihm die Tränen über das Gesicht. Ich dachte nur: , Was ist das denn jetzt?, und dann hat er mir nachher erklärt, er war so froh, dass da eine strahlende, reifere Frau reinkam, (lachend) und kein junges Ding und keine Nonne." (Interview 5, S. 24/28)

Die Befragte des Interviews 5 spricht die Vorbehalte an, die ihr nach der Sterbebegleitung ihres Sohnes später als ehrenamtliches Mitglied eines ambulanten Hospizdienstes entgegengebracht wurden. Sie beschreibt die negative Erwartung des Besuchten an den Hospizdienst und seine Befürchtung, dass er von jemandem besucht würde, der aufgrund der Religiosität, des Geschlechts, des Alters oder der Lebensform nicht seine lebensweltlichen Erfahrungen teilen und nicht entsprechend seinen Bedürfnissen mit ihm kommunizieren könne.

\section{Schlussbemerkung}

Während der Vorbereitung und der Wahl einer geeigneten Erhebungsmethode, im Verlauf der Interviews selbst und in den sich wiederholenden Auswertungsphasen der Transkripte wirkt die Intimität des Erzählens vom Sterben nach. Diese Intimität prägt das Erinnerte und den Stil des Sprechens und drückt sich nicht zuletzt in dem großen Umfang des gewonnenen Untersuchungsmaterials aus.

Die Erfahrung des Todes anderer Menschen ist eine "Grenzsituation par excellence und eine Bedrohung der "Gewißheit der 
Wirklichkeiten «" des Alltagslebens. Krisenerfahrungen, insbesondere der Tod und das Sterben von signifikanten Anderen, heben die Selbstverständlichkeiten der Lebenswelt auf..$^{10}$ Die Zuverlässigkeit von Personen und Objekten, Routinen und Gewohnheiten sind Bestandteile einer möglichen Selbstvergewisserung unseres Selbst in der Interaktion mit anderen. ${ }^{11}$ In Anlehnung an Giddens ${ }^{12}$ kann formuliert werden, dass der Tod diesen "protective cocoon", der durch die Routinen der Lebensführung aufrechterhalten wird, erschüttert und existentielle Identitätskrisen verursacht.

In jedem Interview stellt sich daher die Furcht vor der Sterbeerzählung in jeweils unterschiedlicher Form als eine Hürde dar. Ist diese einmal genommen, widmen sich die Befragten jedoch vollkommen dem Versuch, ihre Erfahrungen mit dem Sterbeprozess ihrer Angehörigen angemessen in Worte zu fassen. Sie unterscheiden zwischen der erlebten Sterbesituation, ihren Gefühlen, ihren Erinnerungen und sind in der Lage, über ihre Erlebnisse zu sprechen, auch dann, wenn sich deren Relevanz überhaupt erst in der Situation des Interviews herauskristallisiert.

Vor dem Hintergrund der durch die Sterbeerfahrung ausgelösten existentiellen Verunsicherung sind auch die kognitiven und emotionalen Schwierigkeiten der Befragten, ihre Sterbeerzählung zu entwickeln, nachvollziehbar. Die Beschreibung des Sterbeprozesses einer nahestehenden Person lässt die erfahrene Erschütterung der vormals wahrgenommenen Sicherheit sozialer Beziehungen mit den Verstorbenen in jeder weiteren Erzählung wieder aufleben. Über das Sterben nahestehender Personen zu erzählen bedeutet zwar, für sich eine Sterbeerzählung entwickelt zu haben. Es bedeutet aber gleichzeitig noch nicht, ein dem gesellschaftlichen Ordnungsprinzip angemessenes Erzählen zu entwickeln. Dieser Disparität ihrer jeweiligen Sprech- und Gesprächsstile, abhängig von dem jeweils unterschiedlich erlebten Sterben, sind sich die Befragten bewusst. Dies kann die Themen der Sterbeerzählung, wie z.B. Entkörperlichung, Persönlichkeitsbeschreibungen und Wunsch nach sozialer Unterstützung erklären. Die Themenwahl verläuft für die Befragten unbewusst und verweist über das eigent-

9 Peter L. Berger/Thomas Luckmann, Die gesellschaftliche Konstruktion der Wirklichkeit, Frankfurt a.M. 2004, 108.

${ }^{10}$ Alfred Schütz/Thomas Luckmann, Strukturen der Lebenswelt, Bd. 2, Frankfurt a. M. 1979.

${ }^{11} \mathrm{Vgl}$.Anthony Giddens, Modernity and self identity. Self and society in late modern age, Cambridge 1991.

12 Ebd., 129. 
liche Erinnern und die daran gekoppelte Bedeutungszuschreibung - und zudem auf eine narrative Rückgewinnung von Sicherheit, die die Sterbeerzählung im Zuge des Interviews überhaupt erst ermöglicht.

- Michaela Thönnes, M.A., ist wissenschaftliche Mitarbeiterin und Lehrbeauftragte am Soziologischen Institut der Universität Zürich. Die Analyse soziologischer Aspekte des Sterbens bildet ihren Forschungsschwerpunkt. 